\title{
El mundo no es dueño en su propia casa: hacia una teoría-narrativa y alternativa de la globalización
}

\author{
RUbÉN DARÍo García EscobaR*
}

\section{RESUMEN}

Dos hechos caracterizan la globalización: de un lado, el incremento de interacciones sociales ha dado lugar a un nuevo ámbito social que se caracteriza por tener sus propias instituciones e imaginarios sociales, los cuales se superponen o entran en conflicto con aquellos que son propios de sociedades particulares; de otro lado, este nuevo ámbito social cuenta con sus propias clausuras, tendencia de lo social a explicarse a partir de sí misma. Juntos hacen parte de un mismo fenómeno por el cual el planeta entero ha participado de lo que en este trabajo se denomina clausura mundánica, proceso mediante el cual el mundo deviene sujeto-objeto-contenedor, llevándolo a ser un imaginario incuestionable. Precisamente porque nadie duda del mundo es necesario hacerlo y analizar cómo se manifiesta, qué efecto tiene en la experiencia de vivir en un ámbito social global, unitario e interdependiente.

Para ello el artículo se encuentra dividido en tres partes: primero, análisis de la mundialización como el proceso por el cual el mundo se hace sujeto de su propio devenir; segundo, análisis de la globalización como el proceso mediante el cual el mundo logra coincidir con la tierra o consigo mismo; tercero, siendo los dos momentos constitutivos de la clausura mundánica, globalización y mundialización han derivado en una tiranía de mundo que no es más que la situación de heteronomía a la que conduce el mundo en la forma de una alienación frente a los entes y el tiempo. En el artículo se exploran las propuestas que caracterizaron el ánimo intelectivo de comienzos del siglo $X X$ y, también, se acudirá a ciertos aportes de la Escuela Inglesa de Relaciones Internacionales por ayudar a enriquecer esta discusión. El objetivo de esta indagación es poder elucidar ciertos elementos que permitan construir una narrativa alternativa que pueda ayudar a comprender mejor la experiencia de vivir en un mundo en el cual el neoliberalismo se ha hecho una razón global.

\section{Palabras clave}

Globalización; Relaciones Internacionales; Filosofía; Política mundial; mundo; institución; clausura.

\section{TITLE}

The world is no longer the master of its house:towards a narrative-theory and alternative of globalization

\section{Extended Abstract}

The current socio-historical situation is characterized by the fact that nobody doubts the World. What does this mean? Through a simultaneous process of mundialization and globalization theWorld has become a social imaginary shared by the biggest part of humanity. This has been possible by what is called in this article mondanic closing, the phenomenon through which the world has become its own subject. This is the result of a longue durée process which began in the intermediate period between the Renaissance and the Enlightenment. During this period it was plausible and desirable for some people to get access to the entire planet to fulfil personal and political goals, leading to an increase in the movement of people, objects and ideas.

This process of expansion gave rise to the mondanic closing. In the context of this analysis, closing is understood as the tendency though which each society or social field explains itself departing from its own social imaginaries as an extrasocial force; in other worlds, closing is the fact of social auto-reference and society's tendency to explain itself as the result or cause of an extra-social force. The mondanic closing is composed of two moments: first, the moment of the world as

* Rubén Darío GARCÍA ESCOBAR, Magíster en Relaciones Internacionales $y$ estudios políticos del Colegio Mayor de Nuestra Señora del Rosario; candidato para Magister en Filosofía de la Pontificia Universidad Javeriana. Se ha desempeñado como consultor y educador para el sector privado en temas de cuidado, autocuidado, filosofía del cuidado, política y filosofía política. Es miembro activo del grupo de investigación "Subjetividad y pensamiento crítico" del Departamento de Filosofía de la Pontificia Universidad Javeriana y miembro del comité directivo de ReDecolat, plataforma independiente de difusión de conocimientos alternativos. institucional. Contacto: garcia. es.ruben@gmail.com

Recibido:

09.03.2021

Aceptado: 05.07.202।

DOI:

https://doi.org// 0.15366/relacionesinternacionales202I.48.004

Formato de citación recomendado:

GARCÍA ESCOBAR, Rubén Darío (202I). "El mundo no es dueño en su propia casa: hacia una teoría-narrativa y alternativa de la globalización”, Relaciones Internacionales, $n^{\circ}$ 48, pp. 67-83. 
subject-object-container, through this contradictory, complementary and superimposed understanding of the world, it is simultaneously the object of a rationality of control, and it is the subject of its own becoming; second, the moment of the becoming of victim-victimizer-of-itself, the world's logic of expansion makes possible the idea of an interdependent and unitary world, but at the same time this world is fragile through its own means of expansion which involves an economy of exploitation and the development of a never-ending war industry.

These two moments help to understand, as an alternative narrative of globalization, the becoming of a unitary and interdependent world process in which intellectual and material changes collide. This has been a violent process and, at the same time, this same process increases the violence. By way of the closing, being-in-the-world is characterized by the loss of the tranquility, it is to say that the day to day living is no longer tranquil and security becomes a central value. On the other hand, being-in -the-world is experienced as being in a social field that is hostile because the world, which we usually think of as controllable, shows itself every day as a force that cannot be controlled.This is reflected in the lack of ground or stability characterizing world politics and the overall dimensions of existence as well, a life full of objects and services related with security, safety and care.

Through the becoming of the mondanic closing and thanks to it, a new social field has emerged: the field of the global. Although inter-cultural relationships existed before the beginning of globalization, this new field is characterized by the way in which the mondanic closing has informed the institutional framework and the primary social imaginaries that make the "global" possible; particularly, the predominance of state and market as the main institutional instances. They make it possible to experience the world as a unitary and interdependent field of action: on the one hand, because they made the West's expansion throughout the planet possible and ensured the processes of institutionalization of social imaginaries in colonized societies; on the other hand, because state and market became the only possibility of dominated societies to be independent and be recognized by its western counterparts. For this reason, market expansion and state building are just two sides of the same phenomenon. They are not independent, as is assumed by erroneous formulations which situate state and market as contradictory institutions with different goals.

In this way, the main manifestation of the mondanic closing is world tyranny.This refers to a social existence in which humans are alienated from the world in the form of being and time. Tyranny is understood in the way Plato conceives it in Republic, as a form of government and a shape of the soul characterized by an existence uncontrolled, a non-sense and a non-being. Something similar happens with the world. Considering its functioning and the imaginaries that make progress and personal fulfillment the main goals of human reality, it is possible to state that the becoming of the world is the possibility of human realization. But like the tyrant in Plato, this is just an illusion. The world is not the master in its own house, it is the victim of its own means.Warfare makes it fragile, putting it at the edge of the abyss; material interdependence puts a pressure on nature that is not possible to sustain in the long run; wealth needs a more and more unequal economic system and the development of political apparatuses of oppression. In other words, the world is its own victim. In order to accomplish this objective, the article is divided into three parts: first, there is an analysis of mundialization as the process through which the world has become the subject of its own happening; second, I turn to an analysis of globalization as the process by which the world has coincided with the earth or with itself; third, being constitutive moments of the mondanic closing, I show how mundialization and globalization have configured a world tyranny, which is the shape of an existence alienated by being and time.

Finally, to address the problematic of the mondanic closing, this article turns to philosophy and International Relations at the same time, considering that an integral approach is necessary. In the first place, philosophy gives an important insight because the World has been one of the main preoccupations of this field since the Enlightenment and in the XX century it arrived at a new perspective of this problem. For this reason, the article resorts to some common areas of phenomenology, particularly the concept of world and facticity. In the second place, International Relations suits this analysis because it is one of the results of the closing and the largest part of its production and research is oriented toward the problem of theWorld as a social field based on unity and interdependence.The English School of International Relations, despite some of the critics of its Western orientation, is useful to understand the world as founded on a common institutional framework which makes experience of the World possible.

\section{KeYWORDS}

Globalization; International Relations; Philosophy;World Politics;World; Institution; Closing. 


\section{ntroducción}

El mundo no siempre estuvo ahí. Esto lo sabemos y por ello las últimas décadas se han caracterizado por la construcción de una conciencia histórica que permita dar cuenta de ello (Augé, 2006; Nancy, 1997; Sloterdijk, 2010). Los esfuerzos por construir una narrativa del mundo han girado en torno a dos ejes centrales: de un lado la globalización, como aquella transformación espacio temporal que estrecharía las distancias entre diferentes culturas y permitiría el incremento de relaciones sociales entre distintos grupos humanos (Reder, 2012, p. I5); de otro lado el colonialismo, como aquel proceso expansivo de una forma social a lo largo y ancho del planeta, que ha devenido en el predominio de ciertas estructuras sociales (Grosfoguel, 2016). En las valoraciones e interpretaciones sobre estos ejes se manifiestan disputas geopolíticas y culturales que hunden sus raíces en las concordias y desavenencias históricas que han resultado del proceso por el cual el mundo ha llegado a ser uno solo. En medio de esta búsqueda por establecer la historicidad del mundo y de nuestra presencia en él, este se ha hecho un fenómeno del cual ya nadie duda. Pero precisamente por constituirse como un hecho indudable se hace necesario e ineludible indagar sobre él.

Partiendo de este escenario, este artículo indaga algunos elementos iniciales para la elaboración de una narrativa sobre el mundo actual que permita, desde un enfoque filosófico, indagar en la experiencia de estar-en un mundo globalizado.Ya existen muchas interpretaciones de la globalización desde la filosofía como es el caso de Rede, Derrida, Hardt y Negri, Sloterdijk, Dardot y Laval, Dussel, entre otros. Pero el enfoque diferencial de este espacio consiste en indagar sobre el fenómeno de mundo, el cual está constituido a partir de la dinámica social que será llamada clausura mundánica, proceso por el cual el mundo ha devenido sujeto de su propia mundialización y ha llegado a coincidir con la tierra para formar el globo (globalización). El objetivo central es elucidar el mundo global, fenómeno que a día de hoy supone un fundamento incuestionable de la realidad humana (Pineda, 20I3).

Para ello, este espacio estará dividido en tres partes: primero, esclarecimiento de la forma en que se está entendiendo el fenómeno del mundo y en qué consiste el proceso de mundialización como una momento fundamental de la clausura mundánica; segundo, se aclarará el contenido de la globalización a la luz del concepto de clausura y su relación con la mundialización como un correlato de escala; finalmente, se plantean algunos elementos iniciales de la experiencia de un mundo globalizado, específicamente: la intranquilidad, lo imprescindible y la tiranía del mundo. El objetivo no es sentar de una vez por todas una nueva narrativa o historia de la globalización del mundo contemporáneo, sino comenzar a explorar los elementos iniciales que puedan conducir a ello a partir del análisis de la experiencia del estar-en o ser-en-situación, que busca responder a la necesaria problemática de la convivencia (Sloterdijk, 2010, p. 23). La metodología que se siguió fue la de una revisión exhaustiva y amplia de textos que se enfocaran en dos temáticas: aportes que permitieran dar cuenta del ánimo intelectual y cultural que informa la concepción actual del mundo; aportes en el ámbito de la historia enfocados en lecturas alternativas del proceso de globalización. 


\section{La mundialización: cierres y aperturas de la experiencia de estar}

A lo largo de la últimas décadas se han develado sucesos constitutivos de la historia de la globalización que habrían sido ignorados o pasados por alto (Bonialian, 20 I8; Dussel, 1992; Hausberger, 2018; Román, 2018; Santos-Herceg, 2010). Por cuenta de la revisión de los acontecimientos históricos y de los patrones estructurales que le subyacen, han comenzado a emerger nuevas narrativas que han (re)pensado los límites del proceso de globalización. Dos de los casos más significativos son la reconstrucción histórica llevada a cabo por la Escuela Inglesa y que ha indagado en los antecedentes sociales de globalización, haciendo énfasis en la constitución de un ámbito institucional y social global (Buzan, 2014; Reus-Smit y Dunne, 2017);y, de otro lado, aquella emprendida por los enfoques decoloniales, los cuales han cuestionado la espontaneidad y los presupuestos esencialistas del proceso de globalización (Grosfoguel, 2016; Mignolo, 2011; Shahar, 2019). En ambos casos se plantea una transformación de la comprensión temporal del fenómeno de la globalización.

No es la intención de este espacio establecer una historia de la globalización, pero para dar inicio a la indagación pretendida es necesario aclarar algunos elementos del escenario histórico en el que se comprenderá el fenómeno del mundo: lo que se conoce como "mundo" se entiende como el resultado de una serie de trasformaciones del marco existencial europeo que inició aproximadamente a partir del siglo XV y el cual se constituirá en uno de los momentos centrales de la experiencia de la globalización (Pineda, 2013); desde este momento, en que comienza la expansión de occidente, la idea de mundo se inserta en el imaginario colectivo de distintas sociedades, dando lugar a una valoración fáctica y normativa de la plausibilidad y necesidad de emprender un proyecto que solo sería pleno en tanto se expandiera a lo largo de la tierra o al menos contara con ella (Bonialian, 2018; Miró-Quesada, 2018); de estas transformaciones iniciaría un proceso que aún no se ha detenido y el cual resultaría en la asimilación por buena parte de los habitantes de la tierra de la unidad-mundo (Reder, 20I2, p. 40) o lo que Sloterdijk (20I0, p. 22) ha denominado como "monogeísmo", la creencia en la unicidad del mundo y la cual va más allá de la simple idea de contigüidad de lo espacio-temporal.

En este análisis nos adherimos a aquella interpretación histórica que identifica la globalización como un proceso de larga duración (Hausberger, 2018), el cual ha logrado prolongarse hasta el presente debido a que ha establecido un ámbito social que se sostiene gracias a un complejo institucional, que va más allá de las organizaciones económicas que han dirigido la etapa más reciente del proceso, lectura de la cual Stiglitz (2002) es una de la figuras más representativas. Este desarrollo sociohistórico informa y es informado por lo que se denomina como clausura mundánica, proceso que se puede dividir, con fines analíticos, como teniendo dos momentos constitutivos: de una lado la mundialización y de otro lado la globalización. El primero de ellos será el tratado en este apartado y luego se pasará al siguiente, sin que esta división pretenda dar mayor relevancia a uno u otro.

Primero, será necesario esclarecer el modo en que se entiende el mundo y cómo es que este lleva a una clausura. El concepto de mundo ha tenido dos momentos fundamentales. El primero de ellos tiene que ver con su emergencia como objeto de indagación filosófica y científica en el marco del Renacimiento y posteriormente en la llustración. Durante este periodo hay una superposición de lo real y lo falso en un mismo plano, el mundo real viene a las personas y solo 
es cuestión de la razón y de la técnica para develar la verdad en él (Berman, 20 I , p. II3). Este es el mundo que se entiende como objeto de control, accesible en su totalidad y que es una herramienta más para la realización de los hombres (Kleber, 2018). El segundo momento, emerge de la mano con la fenomenología en auge desde inicios del siglo $\mathrm{XX}$ y entiende el mundo como uno de los momentos cooriginarios de la existencia, una estructura que determina el estar-en como el modo existentivo de lo humano y el cual supone una totalidad de sentido (Heidegger, 1997, p. 68; Nancy, 2002, p. 34). De un lado, el mundo es entendido como un ente totalmente accesible y controlable; de otro, el mundo es un fenómeno que está en el centro de la existencia humana y cuya comprensión se nos escapa entre los dedos por estar inmersos en él.

La hipótesis de este artículo postula que estas dos formas de comprender el mundo remiten al fenómeno de la clausura mundánica del cual son, a un mismo tiempo, partes constitutivas. A lo largo de la expansión de la idea de mundo, de su inserción en el imaginario colectivo, se ha desarrollado una totalidad heterogénea, llena de conflictos, contradicciones e irregularidades, en la que se enfrentan las nociones del mundo como sujeto y como objeto, parte de las personas o separadas de ellas, como esclavo/herramienta o amo. A pesar de que los análisis tienden a favorecer alguna de estas posturas, lo cierto es que la una no puede ser comprendida sin la otra y en el conflicto al que dan lugar se constituye un sentido que clausura el ámbito histórico social del mundo globalizado actual. La clausura es una "tendencia" de lo social que se da en el proceso de donación de sentido, la cual consiste en que la sociedad comienza a explicarse únicamente a partir de significaciones cerradas que le son propias, dando lugar a una situación heterónoma en la que la sociedad se entiende a sí misma como el resultado de una fuerza extra social (Castoriadis, 1988, 2007).

La clausura mundánica no es propia de una sociedad particular. Este es un fenómeno que incumbe a todas las sociedades que se han visto involucradas en el proceso de expansión de un marco existencial caracterizado por la unidad y la interdependencia. El primer momento de esta clausura corresponde a la "mundialización" y “mundanización”, momento de la formación-mundo y del devenir-mundo; es el mundo abandonando su estatus de objeto para devenir sí mismo, su propia mundialidad (mondialite) (Nancy, 2002, p. 33). Parte importante de la modernidad consistió en hacer del mundo un ente, un objeto-contenedor de objetos, a través de la clasificación, la diferenciación y el relacionamiento; revelar las leyes subyacentes de su funcionamiento y, una vez adquirido el conocimiento de estas leyes, manipularlo (Bauman, 1998; Castoriadis, 2007). En otras palabras, descubrir la verdad para hacer la verdad.

Esta dinámica dio lugar a la actualmente criticada diferenciación sujeto-objeto que constituye la subjetividad moderna, en términos de una relación de dominio del primero sobre el segundo. Sin embargo, la construcción del mundo-objeto se dio de manera simultánea con la del mundosujeto. Si bien existían unas leyes concretas y a partir de su conocimiento se podía desplegar una racionalidad de dominio, al mismo tiempo, en la medida en que el mundo se revelaba o se negaba a hacerlo, se constituyó en una fuerza extra social: el mundo devino autónomo. El imaginario de control total y racional del mundo tiene un papel fundamental, pero también el imaginario de la incontrolabilidad (autonomía) del mundo, lo cual ha derivado en el papel privilegiado que tiene la seguridad y la protección en el mundo actual (Beck, 2008; Sanahuja, 2019). El desnudamiento de la realidad humana resultó en la intensificación de la experiencia de peligro y riesgo. El mundo está 
lleno de posibilidades para los proyectos humanos, pero a un mismo tiempo es la amenaza latente de que detrás de cada una de sus leyes aparezca el sufrimiento, el dolor, la enfermedad, la muerte, y nada se puede hacer al respecto.

La naturaleza, la idoneidad de los entes, sería la principal herramienta que aseguraría la autonomía del mundo, su interdependencia y unidad. Por ejemplo, bajo el imaginario social de naturaleza se establecería la unidad de la humanidad. Esto supondría un movimiento que se encargó de declarar y divulgar un discurso de derechos sustentado en el respeto y la dignidad humana, de acuerdo a una comprensión concreta de la naturaleza-esencia de cada persona (Kim, 2017; Taylor, 2006, p. 40). De otro lado, permitiría la unidad de la humanidad como objeto de las leyes del mundo, parte de las cosas que "contiene" y que malea indefinidamente (Bauman, 1998, p. 18). De esta manera la naturaleza se convirtió, de un lado, en uno de los ejes centrales de la indagación científica y filosófica $y$, de otro lado, en una herramienta política de primer orden. El imaginario primario de naturaleza permitió constituir algunos de los corpus políticos más importantes como fue el caso de Hobbes y Rousseau (Evcan, 2019) y está en el seno mismo del capitalismo tal cual se presenta en la actualidad (Foucault, 2007; Pignol, 2017). Una de las formas de entender la interdependencia en el mundo actual es como la fragilidad y dependencia de ser tocado por la naturaleza del otro, su impulso al gozo, al poder, en fin, a la anarquía.

El mundo, en su faceta de objeto-contenedor, no solo contiene los entes como tal o es médium para que estos lleguen a ser. El mundo es contenedor de esencias, de naturalezas y de los imperceptibles procesos que las sostienen. Estas dos facetas se revelaron en el proceso de constitución de un ámbito social global a través de la colonización y la consolidación del estado y el mercado en Europa (Coca y Baltos, 2020; O'Hagan, 2017). En particular la esencia del mercado, como lo sostendrían Marx y Engels (1973), es expansiva y la del estado es la de modular la tendencias expansivas, propias y las de otros estados o cuerpos políticos (Cohen, 1984). Juntos, mercado y estado, serían entonces los encargados de modular, controlar y disponer de las personas y las cosas para garantizar un nuevo ámbito social que era necesario para poder alcanzar los fines supuestos por el imaginario colectivo: el progreso, el equilibrio económico, el orden político, entre otros. Es así como se llega a la representación del ámbito social/global como anárquico.

La anarquía internacional refleja muy bien la contradicción entre la dimensión del mundoobjeto y la del mundo-sujeto, cuya esencia-contenido es la de contener las esencias de los entes particulares. De un lado, el ámbito internacional es el resultado de la racionalidad de dominio implementada sobre la materia-mundo para dar lugar a un orden político y garantizar a lo largo de la tierra el progreso histórico. Tal fue la empresa capitalista, colonial e imperial vista desde Europa (Grosfoguel, 2016; Nancy, 2002; Santos-Herceg, 2010), pero también la de aquellas personas que desde la periferia se unieron a tal proyecto (Hausberger, 2018). De otro lado, ante la ausencia de un ordenador político, moral y jurídico, y las dificultades que emergían en medio de un proceso expansivo violento lleno de resistencias y luchas, el mundo del sistema internacional se constituyó como un ámbito social violento, peligroso, lleno de amenazas e impredecible (Christov, 2017; Sjoberg, 2017). El mundo que contenía y dominaba la humanidad no era más que la prolongación de una naturaleza humana nefasta, proclive a hacer daño. Esta tensión del mundo-contenedor de lo internacional o global sigue manifestándose con toda intensidad a día de hoy. 
Este proceso sociohistórico corresponde a la mundialización, una de las dimensiones de la clausura mundánica. La mundialización es el proceso por el cual el mundo se transformó en una fuerza extra social, la cual está dada de una vez y por todas, constituyéndose como el determinante primario de la existencia humana. Desde esa distancia infranqueable que le ubica como sujetoobjeto-contenedor-de-esencias, el mundo es el creador de lo humano, el primer artesano de la subjetividad y el principal legislador de la sociedad. Desde esta perspectiva, la historia de la humanidad no es el resultado de la creación en el campo histórico social, sino el simple devenir del mundo, su entrada en plenitud y la forma en que contribuimos a dicha realización. Todos trabajamos para llevar el mundo a su fin(al). Contrario a lo que se sustenta en buena parte de la crítica sobre la relación mundo/persona, como una que se da únicamente en términos de dominio (Grosfoguel, 20 16; Slater, 2008), el fantasma del des-control y el des-orden sigue presente, hasta el punto de que las personas han sido reducidas a ser solo materia maleable frente al mundo: "todos esos descubrimientos, todas esas investigaciones, solo persiguen una cosa: hacer admitir al hombre que no es nada, absolutamente nada" (Fanon, 2009, p. 52).

\section{Globalización: la coincidencia del mundo consigo mismo}

La mundialización, el devenir del mundo en sujeto, no puede ser entendida sin el correlato de su expansión: el mundo es mundo porque tiene la pretensión de estar en todo tiempo y lugar. Lo que permite entender la globalización como una transformación espaciotemporal es el impacto que resulta de ciertas transformaciones sociohistóricas que alimentaron y se vieron alimentadas por un proceso que afectó la forma de percibir y vivenciar el mundo. En primera instancia, desde el Renacimiento comenzó a gestarse la posibilidad, viabilidad y necesidad de llevar a cabo exploraciones (Dussel, 1992; Santos-Herceg, 2010; Taussig, 2002), en un pequeño grupo de personas que luego terminarían por dar lugar a la subjetividad dominante del aventureroemprendedor y serían quienes llevarían a cabo la "revolución del espacio" que caracterizó la globalización terrestre, la cual empezó en el periodo de transición entre el Renacimiento y la Ilustración (Sloterdijk, 2010, pp. 65-67).

En la medida en que los proyectos exploratorios se desarrollaban, en medio de éxitos y fracasos, el mundo fue expandiéndose a lo largo de la tierra y se constituyó como el espacio vital de las sociedades del momento. La coincidencia del mundo con la tierra era necesaria para alimentar los imperativos imperiales, para dar vitalidad al comercio, tener acceso a fuentes de materias primas y a nuevos mercados para alimentar las industrias nacionales (Hausberger, 2018; Lascurain, 2017, p. 25; Mignolo, 20I I); es decir, la coincidencia del mundo con la tierra era la condición de posibilidad del proyecto moderno, de poder alcanzar al orden político y social al que aspiraba. Tal coincidencia no solo garantizó la inserción del imaginario social del mundo como sujeto-objeto-contenedor en diferentes sociedades, sino que instituyó un nuevo espacio social, necesario para que emergiera la experiencia y, posteriormente, el lenguaje de lo global. Tal comprensión se encontraba, por ejemplo, en las obras de Kant o Hegel: para el primero, por lo menos en La paz perpetua, el mercado haría las veces de garante del orden social y este mercado era siempre entendido en clave de mundialidad; para el segundo, en la Fenomenología del espíritu, el devenir de la historia no era más que la "evolución" o proceso dialectico tras el cual el mundo 
coincidiría consigo mismo, sería espíritu.

Más allá de la pretensión de universalismo que caracterizó la filosofía y la ciencia durante la llustración (Bauman, 1998;Taylor, 2004), que no fue la única tendencia del momento (Hardt y Negri, 2002), lo que marcó este periodo fue el hecho de que el ideal de orden y progreso contaba e involucraba la coincidencia del mundo con la tierra, la unidad de las totalidades sociales. Por ejemplo, durante la colonización las potencias necesitaban de un mundo entero para conquistar nuevas tierras, productos y personas, para poder suplir sus proyectos internos de construcción estatal (Grosfoguel, 2016; Nancy, 2002). En la actualidad esta perspectiva se ha generalizado, cada proyecto estatal cuenta con que el mundo esté repleto de estados capaces de garantizar la seguridad mundial para permitir el adecuado desarrollo de la economía global (García, 20l7). Los lenguajes de las ciencias y de la ontología moral universalista serían los ejes de la constitución de este ámbito social global.

La globalización suele ser entendida como un proceso económico y cultural que se desarrolló al margen de las regulaciones existentes y que llevó a que se desregularizaran las relaciones interculturales. Pero lo cierto es que el estado tuvo, y tiene, un papel fundamental en este proceso y, en particular, en su relación con el capitalismo. En primera instancia el estado es el "hacedor de guerra" que garantiza el control territorial y la extracción de recursos (Cohen, I 984; Tilly, 2007), pero también es la forma social común a distintas sociedades que es capaz de garantizar la coincidencia del mundo con la tierra: desde el siglo $X X$ el mundo está atestado de estados que ejercen o buscan ejercer un dominio sobre una población y un territorio (Ayoob, 1995). La prevalencia de dicha forma sociopolítica es lo que daría lugar a la Sociedad de Estados (Buzan, 2014; Reus-Smit y Dunne, 2017). Si bien, el progreso económico era la significación que alimentaba las expectativas por lograr el bienestar y el orden, el estado era el garante de dicho progreso, de la posibilidad de disfrutar el fruto del trabajo, de no ser destruido por otras sociedades y, por el contrario, ser reconocido por ellas.

Al comprender la guerra como "el gran negocio", Fanon (I975) logró dar con el punto en que se entrecruzan estado y mercado, como dos dimensiones fundamentales, complementarias, heterogéneas y contradictorias de la mundialización y la globalización: "frente a una nueva unión impuesta, no propuesta sino afirmada, pesan con todo su peso los cañones y los sables" (p. $41)$. Aunque no se les puede adjudicar la dirección del devenir del mundo, proceso demasiado amplio para ser reducido a dos instancias institucionales, estado y mercado tendrían un papel privilegiado en el marco de la clausura mundánica: I) permitirían la participación autónoma de sociedades particulares en las dinámicas globales, con la garantía de mantener la seguridad gracias a un aparato militar masivo (Cohen, 1984), es decir, la supervivencia y el progreso en medio de la anarquía; 2) una vez establecida la paridad por medio de la violencia, se asegura la participación en el ámbito global para acceder a los dividendos de la historia y el progreso; 3) estado y mercado, al ejercer la violencia sobre poblaciones y territorios, aseguraban la asimilación de imaginarios sociales que hacían a las personas proclives a la reproducción de estas instituciones.

Estos tres aspectos se superponen unos a otros en unos ciclos (re)productivos que concluyeron la coincidencia del mundo con la tierra. Primero, el ciclo violencia y mercado: la economía necesita del mundo entero para poder realizarse plenamente, lo que garantiza a una 
sociedad el acceso autónomo y seguro a esta plenitud es la posesión de una maquinaria de guerra y, a su vez, esta puede abastecerse en la medida en que la sociedad progresa económicamente. Segundo, el ciclo de la violencia instituida e instituyente del mundo: la guerra y las diferentes formas de violencia suponen el desarrollo de una forma de Derecho Internacional que regula las relaciones entre los estados y garantiza derechos individuales (Dunne, 200 I; Kim, 20I7; Pérez de Armiño, 2007) y tiene que ver con el desarrollo de un ámbito metasocial institucional en el cual se establecen lenguajes, técnicas, formas de hacer cosas y de hacer frente a ellas (Castoriadis, 1988). La Escuela Inglesa ha sido muy acertada al resaltar la constitución de este ámbito social, su carácter histórico y las relaciones sistémicas y societales que se desarrollan en él (Buzan, 1993, 2014; Tepecikliolu, 2016). Sin embargo, ha pasado por alto, al menos en la obra de sus principales representantes, el papel que ha tenido la violencia como instancia instituyente, al concentrarse en la norma institucional y jurídica.

En la violenta expansión del mundo a lo largo y ancho de la tierra, la violencia organizada ha tenido un papel fundamental en el establecimiento de un orden mundial jerarquizado en torno a líneas de género, clase y raza (Grosfoguel, 2016; Hardt y Negri, 2002; Santos-Herceg, 20I0). También ha sido la principal herramienta instituyente de la que ha dispuesto occidente para garantizar la (re)producción del mundo, mantener su unidad e interdependencia. Esto se manifiesta particularmente en las zonas periféricas que fueron objeto de políticas expansionistas. Por ejemplo, tras el violento proceso de colonización, las élites latinoamericanas decidieron comprometerse con un proyecto burgués ilustrado y, posteriormente, burgués positivista (Bragoni, 2013; MiróQuesada, 1962), que suponía ante todo aceptar al estado y el mercado como las instituciones que permitirían la independencia, la autonomía y el reconocimiento de otras sociedades. La globalización es ante todo una hegemonía que está sustentada en un consenso (Sanahuja, 2019, p. 6I), que dice en primera instancia que el mundo es uno, que no solo está disponible sino que es necesario acceder a él, y que las formas de hacerlo son por medio del estado y el mercado. Salir de esta lógica es lo que supone romper la dinámica del dominio y la violencia (Shahar, 2019).

Si la inauguración del galeón de Manila en I 565 que conectaba a Nueva España con China fue el hito de la clausura material del mundo en la que se "enlazan los dos espacios restantes para la constitución de una red verdaderamente global” (Bonialian, 2018, p. 788), la legitimación del estado y del mercado constituyen la globalización del marco existencial que rige lo social a nivel global, como aquel ámbito en el que el mundo es autónomo, sujeto de sus propios procesos e independiente del hacer humano. En otras palabras, la globalización fue el proceso por el cual se legitima la existencia del mundo como estando ahí en todo momento. De este consenso resulta el carácter pseudorreligioso de la clausura mundánica, es decir, su "carácter de ultimidad y definitividad" (Román, 2018, p. I8), que se manifiesta en un ejercicio del poder heterónomo que puede ser entendido como poder mundial: el poder se constituye siempre y en todo momento como mundial.

Desde esta perspectiva se entiende la globalización como un proceso que iniciaría por cuenta de simultaneas trasformaciones materiales, intelectivas y espirituales entre diferentes sociedades desde las postrimerías del Renacimiento. Este debe entenderse solo en un primer momento como una empresa exclusivamente europea. Una vez los proyectos europeos comienzan a afectar la existencia de diferentes sociedades, dicho proceso se vuelve una empresa común 
que daría lugar a un ámbito metasocial. Los contactos interculturales ya habrían precedido la globalización, dando lugar a circuitos económicos y culturales (Amin, 20I I). Sin embargo, lo que interesa en la globalización y mundialización son los carácteres que toman estos procesos por cuenta de la clausura mundánica, cerrazón intelectiva y material que afectaría la experiencia del estar-en la tierra y, por ende, los procesos de donación de sentido.

\section{La tiranía del mundo}

Una de las grandes hipótesis filosóficas que ha penetrado el imaginario social e influido en los desarrollos de diferentes campos de conocimiento es la facticidad, entendida como el hecho de que el mundo es inescapable e inevitable. Para filósofos como Heidegger, Sartre o Merleau-Ponty la facticidad supone el hecho de que cada uno de nosotros está abandonado a una situación particular, determinada en todas sus antípodas por el mundo, los entes y el tiempo. Entendido de otra manera, la facticidad supone un "siempre demasiado tarde" que se constituye como punto de partida inevitable al momento de hacer frente a la existencia. La comprensión general (hegemónica) de la globalización es la de un proceso que se habría desarrollado a partir de la década de los setenta por cuenta de ciertas transformaciones económicas y tecnológicas que permitirían el incremento de las relaciones sociales. Este se terminaría de concretar tras la finalización de la Guerra Fría en la forma del Neoliberalismo. Desde esta perspectiva, los hitos de la globalización son: de un lado, la emergencia del sistema institucional que daría paso a la conformación de un orden mundial tras los acuerdo de Bretton Woods en 1944 y el Consenso de Washington en 1989 (Arrighi, 2010; Jara, 2019; Sanahuja, 2019; Stiglitz, 2002); de otro lado, la constitución de un marco epistemológico común, el auge de organizaciones no gubernamentales y las nuevas estructuras sociales en red posibles por el internet y otras tecnologías (Hardt y Negri, 2002; Olivé, 2008; Slater, 2008).

De esta lectura, se ha entendido que la "cerrazón" del mundo en torno a ciertos principios que rigen la vida social de casi todas las poblaciones del planeta se concreta hacia finales de la Guerra Fría.

Esto es lo que se ha querido elucidar en las grandes críticas al neoliberalismo y al "pensamiento único" (Bosoer y Leiras, 1999; Ciuffolini, 2017; Laval y Dardot, 2013; Rapoport, 2002). Sin embargo, como todo en el mundo de acuerdo a la facticidad, cuando se proclama, denuncia, apoya y critica esta cerrazón, ya estábamos en medio del "siempre demasiado tarde" de la clausura mundánica. Si el neoliberalismo ha sido posible es porque de tiempo atrás ya nadie dudaba de la existencia del mundo, nadie se sentía capaz de actuar como si pudiera prescindir de él. En su interpretación sobre el impulso intelectivo que prevaleció en Europa en el siglo XIX, Camus (195I) logró intuir la prevalencia del mundo: en el "movimiento de revuelta", momento del sí y el no absoluto, el individuo se pierde en el destino de la especie y el "movimiento eterno de los mundos" (pp. 96); "hay un dios, en efecto, que es el mundo" (p. 98)'. La indagación de este pensador francés apuntaba hacía otros caminos, sin embargo, en lo que concierne a nuestro análisis, es de resaltar el trasfondo de la idea de mundo como clausura.

Texto original : Il y a un dieu, en effet, qui est le monde. 
Desde sus comienzos, el ánimo intelectual y político del siglo $X X$ estuvo marcado por la angustia y la decepción respecto a los proyectos políticos que habían sido emprendidos a lo largo de más de quinientos años de la mano de una racionalidad de control. Fue el momento del resquebrajamiento de la estructura imperial-colonial europea, el estallido de dos guerra mundiales y de las dificultades generadas por las crisis económicas (Gadamer, 2003; Kaufmann, 1960) fue el momento del recrudecimiento de violencias coloniales en zonas periféricas y el incremento de la violencia entre los colonizados por la búsqueda de una respuesta a su situación, el desaliento de seguir siendo lo que eran y no ser reconocidos (Santos-Herceg, 2010, p. I40; Zea, 1978, pp. 274-275, 2010, p. I0). Era el momento de temer ante el tamaño del mundo y a su facticidad.

En la medida en que las transformaciones del marco existencial comenzaron a dar lugar a la experiencia de lo mundial y global como cerrazón intelectiva y cognitiva, el estar en el mundo se comienza a delinear en términos de una vulnerabilidad mundial. La clausura mundánica se caracteriza por ser una forma social que difícilmente puede ser transformada, está fuertemente anclada en la realidad humana. Pero precisamente en virtud de su fortaleza se hace débil, frágil y volátil. Lo que sucede en una plantación de aguacates en México tiene efectos inmediatos e inevitables en el mercado financiero de China o Estados Unidos. Los drones usados por el ejército estadounidense en Yemen para neutralizar objetivos militares generan diversos tipos de respuestas de apoyo o rechazo en diferentes latitudes. Si la primera contradicción constitutiva del mundo es aquella de ser sujeto-objeto-contenedor, la segunda tiene que ver con ser su propio devenir víctima-victimario-de-sí. En la medida en que el mundo coincide con la tierra se hace más frágil. Al buscar hacer frente a esta fragilidad por medio de una racionalidad de control (Bauman, 1998; Beck, 2008), no puede evitar el dañarse a sí mismo. El mundo asume el papel de dios, una dimensión religiosa que fundamenta la existencia social (Román, 2018), pero no es dueño en su propia casa; está a merced de sí mismo, es su propio tirano.

La expansión del mundo se ha sustentado en un modelo imperialista, de expansión territorial y espiritual, de manipulación del cuerpo y la conciencia, que en los últimos años se ha llevado más que nada a través de actores económicos (Abdalla y Faria, 2019). Por cuenta de esto, se ha comenzado a desarrollar un gran debate en torno al estado y el papel que ha desempeñado desde el siglo XX. El lugar común de este debate llama la atención sobre la perdida de relevancia del estado en la definición de un ordenamiento político (Coca y Baltos, 2020; Pérez de Armiño, 2007; Rohbeck, 2018). Este escenario genera sospechas frente a la hipótesis anteriormente planteada de la necesaria relación del mercado y el estado como las instituciones que garantizarían la unidad del mundo, la clausura mundánica. Sin embargo, ante este aparente retroceso del estado hay una forma del mundo que le subyace y el cual puede responder a esta cuestión.

La clausura mundánica, su forma heterónoma y totalizante, ha resultado en una tiranía de mundo que no es otra cosa que aquella ordenación existencial regida por las contradicciones del devenir víctima-victimario-de-sí.A su vez, esta es la tiranía del mundo como totalidad estructurante y remisional, a través de los entes (objetos) y del tiempo. Estar en el mundo es en primera instancia facticidad $y$, por cuenta de ello, también es alienación al tiempo y a los entes. Tanto las fuerzas dominantes, como aquellos contrapoderes que han emergido a lo largo de los últimos cien años se han abandonado irreflexivamente al mundo entendido como totalidad de tiempo y objetos. Por cuenta de esta tiranía ha sido posible la constitución de una sociedad de consumo, que ha hecho 
de esta actividad una institución que jerarquiza (da valor) y dota de sentido (Baudrillard, 20I4; Sánchez, 2004). Pero también en torno a ella se han constituido las principales alternativas políticas y culturales al modelo hegemónico: de un lado, a través de la generalización y totalización a lo largo del planeta de una "conciencia histórica", la existencia se da para la mayoría de personas en términos de estar ocurriendo en el tiempo, irremediablemente atado al pasado y angustiosamente esperando el futuro (Augé, 2006); de otro lado, la forma de buscar el reconocimiento es la de demostrar que se ha estado en el tiempo y la muestra de ello son los objetos (culturales). Por ello la mostración o visibilización ha sido uno de los ejes centrales de la política desde hace un siglo.

Durante la primera parte del siglo XX, el papel del estado fue fundamental por el hecho de ser la garantía de tener un lugar en el mundo. Por ello el auge estatal que se dio tras la finalización de la Segunda Guerra mundial (Ayoob, 1995; Gandásegui, 2007). La tierra, solamente garantizada por el estado, se convirtió en el valor central de la revolución en contra de la colonización (Fanon, 1983, p. 26), la cual permitiría la inserción a la Sociedad de Estados y el reconocimiento de otros miembros. La guerra de liberación nacional, la organización obrera, la lucha guerrillera, el foquismo cubano, entre otros, no fueron más que la expresión del deseo de tener un lugar en medio del mundo a través de la apropiación/construcción de estado. Estos procesos se vieron obstaculizados, dirigidos, manipulados, tanto por élites políticas nacionales a través de procesos de construcción de estado, como por actores externos a través de las intervenciones de orden político y económico promovidas por instituciones internacionales. Esto llevó a que la tierra perdiera la valoración que habría tenido entre los diferentes contrapoderes del orden mundial. Una vez el mundo se ha unido bajo un mismo marco existencial, le fue posible ser propiedad apropiada gracias al estado y el mercado; en otras palabras, tras la clausura mundánica y gracias a ella, todo el mundo, su tiempo y entes, se han hecho susceptibles de ser propiedad y han sido objetos de un gran proceso de apropiación bajo la guía de una razón global neoliberal (Laval y Dardot, 20I5).

Este proceso de apropiación no supone que el estado haya dejado de ser una de las instituciones primarias sobre las que se sostiene el mundo, lugar común incluso entre los defensores del estado que abogan o prevén un regreso a él en los tiempos venideros (Blackwill y Wright, 2020; Erman y Möller, 20 I8). Esto corresponde a una nueva etapa de las ya inquebrantables relaciones entre el estado y el mercado. Sin el estado y la gran maquinaria de guerra de la que dispone, el mundo no podría existir de la forma en que lo ha hecho por más de un siglo, en medio de la clausura. Incluso, durante las décadas de los ochenta y noventa, donde se identifican los principales momentos de retroceso del estado, este seguía teniendo un papel fundamental, aunque no fuera protagónico. El correlato de la gran liberalización económica y política promovida en el marco del neoliberalismo (Cordera Campos, 2017; Keen, 2020; Laval y Dardot, 2013) fue la promoción de la construcción de estado como aquella institución capaz de garantizar el orden y la seguridad mundial: cualquier espacio del mundo carente de estado, de control del territorio y la monopolización del uso de la violencia, constituye una amenaza al orden mundial (García, 2017). Sobre esta idea se basa la emergencia del discurso del estado fallido, vulnerable, santuario o "caldo de cultivo" (Call, 20I I; Flórez, 20I I).

La tiranía de mundo, expresión de la clausura mundánica, es parte del marco referencial de la experiencia de estar en un mundo globalizado, la cual se da a partir de ciertos imaginarios sociales 
comunes como la unidad, la interdependencia, la anarquía y la estructuración en red. Se entiende el concepto de "marco referencial" en el sentido planteado por Taylor (2006, p. 46), es decir, como parte de un conjunto de referencias de carácter normativo y fáctico que funcionan bajo la idea de "lo mejor" en un sentido genérico. Estos marcos son parte de una "ubicación" en el espacio moral a partir de la cual nos identificamos y hacemos en el mundo (p. 5I). El marco de referencia ofrecido por el mundo brinda las herramientas para poder interpretar y valorar nuestro lugar en un planeta globalizado. Este es el que permite que en la búsqueda de una buena vida alguien se plantee la posibilidad de residir en un país distinto al de origen o tratar de imitar un modelo de vida propio de otro contexto. Es decir, no solo plantearse la existencia propia como ocurriendo en el mundo, sino como ocurriendo en un ente sujeto-objeto-contenedor interdependiente y unido: mis posibilidades se extienden por el mundo, “el mundo es mi hogar". Dada la primacía del estado y el mercado, es común que la identidad de la mayoría de las personas en el periodo actual este definida en términos de una relación con tales instituciones: se está o no a favor del estado o del mercado; se compara la vida propia al comparar dos tipos de estados, en el que se vive y en el que es modelo; se valora la vida a partir de la capacidad de consumo que se tenga o del pasado que se persiga.

¿Por qué plantear el fenómeno que se quiere abordar como tiranía? Tal como Platón (1988) mostró en la República, la tiranía y el tirano suponen formas de organización social y una organización del alma impuestas, en las cuales se abría roto todo tipo de orden y equilibrio. Lo que Sócrates logra demostrar en los últimos libros de este diálogo es que el tirano es infeliz, pues aunque en apariencia accede a todo lo que desea y hace todo en orden a su satisfacción, se ha perdido a sí mismo, ha estropeado su alma en un desequilibrio sin-razón y sin-ser (identidad). Algo muy similar sucede con la clausura del mundo, con su tiranía o marco de referencia. Como sujeto-objeto-contenedor, el mundo aparece a la experiencia humana como un ente conocible y controlable (Kleber, 20I8), el cual está al servicio de los mejores “competidores”, que son aquellos que saben las reglas que rigen el mundo, es decir las leyes del estado y el mercado (Keen, 2020).

De otro lado, como devenir victima-victimario-de-sí, el mundo es un lugar que se ha constituido como una amenaza latente que hace que la cotidianidad sea intranquila y el habitar una hostilidad. Los medios de su expansión, las instituciones que lo sostienen y los marcos de referencia a los que da lugar, alimentan los mismos peligros que le amenazan. La búsqueda de la seguridad ha llevado a un escalamiento de la industria militar sin precedentes, la interconexión material genera fragilidades de tipo biológico como la destrucción del medio ambiente o la fácil propagación de pandemias como es el caso del covid-19, el acortamiento de distancias ha servido para mejorar los niveles de vida de distintas poblaciones y a un mismo tiempo son utilizados por el terrorismo global para operar.

El principio totalitario de competencia con que se ha identificado el neoliberalismo (Ciuffolini, 20I7; Han, 20 I4; Laval y Dardot, 20I3) es el reflejo de este desequilibrio. La competencia neoliberal, en la que cada uno es responsable de su propio destino, ha contribuido a que en la experiencia de estar en el mundo prime un principio de ambigüedad de la identidad (Hassanzadeh, 2018; Taylor, 2006, p. 4I), que carece de un sentido que esté más allá de la autorrealización; ambigüedad que homogeniza en una razón neoliberal que se superpone, asimila y rechaza las particularidades que hacen lo humano (Ciuffolini, 2017). Homogeneización no quiere decir otra 
cosa que la ausencia de lugares para existir regidos por otros principios que no sean los del mundo.

En este contexto, y dadas las contradicciones mencionadas, el mundo actual no solo se experimenta como caótico e incontrolable por cuentas de los múltiples riesgos que implica el vivir en un mundo globalizado. El marco de referencia hegemónico todavía nos permite identificarnos como sujetos capaces de ejercer control y abocarnos a un hacer que corresponda con tal vivencia (Keen, 2020). Pero también nos lleva a identificarnos como materia vulnerable y maleable por fuerzas extra-sociales que escapan de nuestras manos.Aunque algunas interpretaciones entiendan el periodo actual como uno de crisis, una problemática relacionada con la gobernanza y la legitimidad (Blackwill yWright, 2020; Sanahuja, 2019, p. 89), esta es la forma normal del mundo, el resultado de su funcionamiento hacia lo que ha establecido como "lo mejor", el cual a un mismo tiempo nos hace sentir seguros y frágiles.

\section{Conclusiones}

A lo largo de este espacio se trató de brindar una interpretación alternativa del mundo a partir de ciertas hipótesis comunes de la filosofía del siglo $X X$, con el objetivo de establecer unos postulados iniciales que permitieran dar lugar a una narrativa alternativa del proceso histórico por el cual la humanidad ha dado lugar a un marco existencial común, total y totalizante, que ha permeado el imaginario colectivo de buena parte de las sociedades existentes. Para ello se analizó el fenómeno de la clausura mundánica, forma en que se conceptualizó el proceso mediante el cual el mundo, ese artificio europeo (Pineda, 20l3), logró constituirse como el fundamento de un marco existencial global que informa los marcos de referencia a partir de los cuales se entiende y valora, fáctica y normativamente, la experiencia de estar en un mundo unido, cerrado e interdependiente. Esta experiencia está constituida por dos momentos de la clausura mundánica heterogéneos, conflictuales, armonizados e interdependientes: I) el ser sujeto-objeto-contenedor, por el cual el mundo simultáneamente se ha autonomizado y constituido como una fuerza extrasocial, y, por otro lado, se hace objeto de dominio de un control racional; 2) el devenir víctimavictimario-de-sí, momento por el cual se trató de elucidar el carácter (auto)destructivo de la tendencia a la expansión del mundo fundamentalmente a través del estado y el mercado.

Se entendió la clausura mundánica como expresándose en una tiranía de mundo. Esta se refiere, en primer lugar, al marco de referencia en el que la vida se sustenta a partir de una alienación frente al tiempo y los objetos; ser es tener historia y objetos que sirvan de evidencia de este "haber pasado". En segundo lugar, remite a la creencia de la plausibilidad y necesidad de actuar de acuerdo a un acceso indetenible, inaplazable e irreductible a todo lo que constituye la existencia para poder alcanzar la realización, la plenitud; es este el sentido profundo de la expansión que caracterizó la mundialización y la globalización. De la misma manera en que Platón entendido la tiranía, se postuló que la tiranía del mundo es un desbalance incontrolable producido por los momentos constitutivos de la clausura y que se entiende como un sin-sentido y sin-razón que da lugar a que se experimente la existencia como una crisis del yo y del orden político, cuando en realidad no es tal. Por cuenta de este contexto, se puede decir del mundo que no es dueño en su propia casa. El devenir del mundo ha sido el devenir de un orden heterónomo que 
se caracteriza por estar siempre más allá de nuestras manos.

Finalmente, lo que se ha buscado con esta exposición ha sido ofrecer un primer elemento para (re)pensar las comprensiones que se tienen de la globalización y de la política mundial. Ciertamente, el concepto de clausura mundánica es insuficiente para explicar todos los complejos y heterogéneos procesos que subyacen a la globalización, sin embargo, es necesario establecer una salida a las comprensiones predominantes y este es el papel que se le ha querido dar a la elucidación de este fenómeno. El proyecto de construcción de una narrativa de la globalización partiendo de la clausura mundánica busca postular una alternativa que haga énfasis en las relaciones que tienen los imaginarios sociales con las formas en que se desea, comprende y se hace en medio de un mundo que en todo momento responde a un ánimo existencial; una narrativa que anteponga la creación y el devenir de lo humano, antes que la heteronomía de significaciones sociales cerradas sobre sí.

\section{Referencias}

Abdalla, M. M., y Faria, A. (2019). Local development versus neoliberal globalization project: Reflecting on marketoriented cities. Revista de Administracao Publica, 53 (I), 84-100. https://doi.org/I0.1590/0034-76 I220170088

Amin, S. (20II). ¿Primavera Árabe? El mundo árabe en la larga duración. El viejo topo.

Arrighi, G. (2010). The long Twentieth Century: money, power, and the origins of our times.Verso.

Augé, M. (2006). Hacia una antropología de los mundos contemporaneos Gedisa.

Ayoob, M. (1995). The Third World Security Predicament. Lynne Rienner.

Baudrillard, J. (2014). La sociedad de consumo: sus mitos, sus estructuras. Siglo XXI.

Bauman, Z. (1998). Modernity and ambivalence. Polity Press.

Beck, U. (2008). La sociedad del riesgo mundial: en busca de la seguridad perdida. Paidós.

Benítez-Gutiérrez, G. (2017). Ciudad digital paradigma de la globalización urbana. Bitacora, 27 (I), 79-88. https://doi. org/https://doi.org//0.15446/bitacora.v27nl.51349

Berman, M. (20I I). Todo lo solido se desvanece en el aire: La experiencia de la modernidad. Siglo XXI.

Blackwill, R. D. y Wright, T. (2020). The End of World Order and American Foreign Policy. Council on Foreign Relations.

Bonialian, M. (2018). Crítica de libro: La globalización temprana. Colegio de México, 2, 785-80I.

Bosoer, F. y Leiras, S. (1999). Posguerra fría, "neodecisionismo" y nueva fase del capitalismo: el alegato del Príncipegobernante en el escenario global de los '90. En Boron,A.y Gambina,J. (Eds.). Tiempos Violentos. Neoliberalismo, Globalización y Desigualdad En América Latina (pp. I07-123). CLACSO.

Bragoni, B. (20/3). La formación de la conciencia burguesa en iberoamérica durante el siglo XIX. En Roig, A. (Ed.). Pensamiento social y polítco iberoamericano (pp. 87-108). Trotta.

Buzan, B. (1993). From International System to International Society: Structural Realism and Regime Theory meet the English School. International Organization, 47 (3), 327-352.

Buzan, B. (2014). An introduction to the Englis School of International Relations: the societal approach. Polity Press.

Call, C. T. (20II). Beyond the "failed state": Toward conceptual alternatives. European Journal of International Relations, 17 (2), 303-326. https://doi.org//0.1177//354066109353/37

Camus, A. (1951). L'homme révolté. Gallimard.

Castoriadis, C. (1988). Lo imaginario: La creación en el dominio histórico-social. En Castoriadis, C. (Ed.). Los dominios del hombre: las encrucijadas del laberinto (pp. 64-77). Gedisa.

Castoriadis, C. (2007). La institución imaginaria de la sociedad. Tusquets.

Christov, T. (2017). The invention of hobbesian anarchy. Journal of International Political Theory, I3 (3), 296-3 I0.

Ciuffolini, M. (2017). La dinámica del neoliberalismo y sus desplazamientos. Para una crítica inmanente en orden a su superación. Studia Politicoe, 40, 85-101.

Coca, C. A. y Baltos, G. (2020). Las dinámicas fronterizas en el siglo XX-XXI: un espejo de la evolución del orden mundial. Relaciones Internacionales, 44, 63-86. https://doi.org/I0.15366/relacionesinternacionales2020.44.004

Cohen, R. (1984).Warfare and state formation:Wars make states and states make wars. En Ferguson, B. (Ed.). Warfare, culture and enviroment (pp. 329-358), Academic Press.

Cordera Campos, R. (20I7). Globalización en crisis; por un desarrollo sostenible. Economía UNAM, I4 (40), 3-12. https://doi.org/10.1016/j.eunam.2017.01.001

Dunne, T. (200I). Sociological investigation: instrumental, legitimist and coercive interpretations of International Society. Millenium Journal, 30 (1), 67-91.

Dussel, E. (1992). 1942 El Encubrimiento Del Otro. Concilium (Estella). 
Erman, E. y Möller, N. (2018). Political legitimacy for our world:Where is political realism going? Journal of Politics, 80 (2), 525-538. https://doi.org/10.1086/694548

Evcan, N. S. (2019). Hobbesian Instinctual Reason versus Rousseau's Instinctual Innocence: Backstage logic of colonial expansions and origin of the left-right political dichotomy. Interventions, 2 I (7), 977-997. https://doi.org/I0.I $080 / 1369801 X .2019 .1585910$

Fanon, F. (1975). Por la revolución africana: escritos políticos. Fondo de cultura económica.

Fanon, F. (1 983). Los condenados de la tierra. Fondo de cultura económica.

Fanon, F. (2009). Piel negra, máscaras blancas. Akal.

Flórez, J. (20I I). ¿Estado o concepto fallido? Problemas que plantea la noción de falla estatal y los índices que intentan medirla. Revista Derecho Del Estado, 0 (27), 193-234.

Foucault, M. (2007). El nacimiento de la biopolítica. Curso en el Collége de France (1978//979). Fondo de cultura económica.

Gadamer, G. H. (2003). Existencialismo y filosofía existencial. En Gadamer, G. H. (Ed.). Los caminos de Heidegger (pp. 15-26). Herder.

Gandásegui, M.(2007).Paradojas de la desconexión: el mundo policéntrico contra el mundo perturbado. En Gandásegui, M.A. (Coord.). Crisis de hegemonía de Estados Unidos (pp. 57-76). Siglo XXI.

García, R. (20I7). Extravagancia estatal: Construcción de Estado e inserción internacional en el siglo XXI, Caso yemen (1990-20I I). Desafios, 29 (2), I27-I68.

Grosfoguel, R. (2016). Caos sistémico, crisis civilizatoria y proyectos decoloniales: pensar más allá del proceso civilizatoria de la modernidad/colonialidad. Tabula Rasa, 25, I53-I74.

Han, B. C. (20I4). Psicopolítica: neoliberalismo y nuevas técnicas del poder. Herder.

Hardt, M. y Negri, A. (2002). Imperio. Paidós.

Hassanzadeh, N. (2018). Race, internationalism, and comparative political theory. Polity, 50 (4), 5I9-546. https://doi. org/10.1086/699754

Hausberger, B. (2018). La globalización temprana. Colegio de México.

Heidegger, M. (1997). Ser y Tiempo. Editorial Universitaria.

Jara,A. (2019). Globalización, transnacionalidad y desprotección de los derechos humanos. Revista Mexicana de Ciencias Políticas y Sociales, 65 (238), 19-47. https://doi.org/ 10.2220I/fcpys.2448492xe.2020.238.65576

Kaufmann,W. (1960). Existentialism: From Dostoevsky to Sartre. Meridian Books.

Keen, S. (2020). El coronavirus expone brutalmente las falacias de la economía neoclásica y la globalización. Revista de Economía Institucional, 22 (43), I7-27. https://doi.org/ I0.1860I/0I245996.v22n43.02

Kim, H. (20I7). Universal Human Rights. En Dunne, T. y Reus-Smith, C. (Eds.). The globalization of International Society (pp. 304-322). Oxford University Press.

Kleber, M. (2018). The metaphysics of globalization in heidegger. Philosophy of Globalization, 369-378. https://doi. org/10.15I5/9783।104924I5-027

Lascurain, M. (2017). Challenges of economic globalization. Revista de Relaciones Internacionales, Estrategia y Seguridad, 12 (I), 23-50.

Laval, C. y Dardot, P. (20I3). La nueva razón de mundo: ensayo sobre la sociedad neoliberal. Gedisa.

Laval, C. y Dardot, P. (20I5). Común: ensayo sobre la revolución en el siglo XXI. Gedisa.

Marx, K. y Engels, F. (1973). Manifiesto del Partido Comunista. En Marx, C. y Engels, F. (Eds.). Obras escogidas: tomo I (pp. 99-140). Progreso.

Mignolo,W. (20I I). The global South and World Dis/Order. Journal of Anthropological Research, 67 (2), I65-I88.

Miró-Quesada, F. (1962). Impacto de la metafísica en la ideología latinoamericana. Cuadernos de Cultura Latinoamericana. Miró-Quesada, F. (2018). El hombre, el mundo, el destino: introducción no convencional a la filosofía. Fondo de Desarrollo. Nancy, J.-L. (1997). The sense of the world. University of Minnesota Press.

Nancy, J.-L. (2002). La création du monde ou de la mondialisation. Galilée.

O'Hagan, J. (20I7). The role of Civilization in the Globalization of International Society. En Dunne, T.y Reus-Smith, C. (Eds.). The globalization of International Society (pp. 185-202). Oxford University Press.

Olivé, L. (2008). La ciencia y la tecnología en la sociedad del conocimiento: ética, política y epistemología. Fondo de cultura económica.

Pérez de Armiño, K. (2007). El concepto y el uso de la seguridad humana: análisis crítico de sus potencialidades y riesgos. Revista CIDOB d'Afers Internacionals, 76, 59-77. https://doi.org/l 0.2424I/revistacidob.vi76.55707

Pignol, C. (2017). Rousseau et la critique de l'économie politique. Cahiers d’Economie Politique, 74, I87-I 96.

Pineda,A. (20I3). Aprés La fin de monde: Critique de la raison apocalyptique. Revista de Filosofia UIS, I2 (I), $222-227$. Platón. (1988). Diálogos IV: Republica. Gredos.

Rapoport, M. (2002). Origenes y actualidad del “pensamiento único.” En Gambina, J. (Ed.). La globalización Económico Financiera. Su impacto en América Latina (pp. 357-363). CLACSO.

Reder, M. (20I2). Globalización y filosofía. Herder.

Reus-Smit, C. y Dunne, T. (20I7). The globalization of International Society. En Dunne, T. y Reus-Smith, C. (Eds.). The globalization of International Society (pp. I8-4I). Oxford University Press.

Rohbeck, J. (2018). Globalización e historia. Diánoia, 63 (80), I I9-I 47.

Román, C.E.(20 I8). Globalization: Chimera and religion. Theologica Xaveriana, I 85 (68), I-29. https://doi.org/ I 0. I I I 44/ javeriana.tx68-185.gqr

Sanahuja, J. (2019). Crisis de la globalización, el regionalismo y el orden liberal: el ascenso mundial del nacionalismo 
y la extrema derecha. Revista Uruguaya de Ciencia Política, 28 (I), 59-95. https://doi.org/ I0.2685 I/rucp.28. I.3 Sánchez, C. (2004). Las máscaras del dinero: el simbolismo social de la riqueza. Anthropos.

Santos-Herceg, J. (20I0). Conflictos de representaciones: América latina como lugar para la filosofia. Fondo de cultura económica.

Shahar. (2019). Fear, Flight, and Freedom: On Anti-colonial Countermasteries and Ontological Insecurities. Horizontes Decoloniales, 5, I65. https://doi.org/10.13169/decohori.5.1.0165

Sjoberg, L. (2017). The invisible structure of anarchy: gender, orders and global politics. Journal of International Political Theory, 13 (3), 325-240.

Slater, D. (2008). Re-pensando la geopolítica del conocimiento: reto a las violaciones imperiales. Tabula Rasa, 8, 335358. https://doi.org/I0.25058/20 I I 2742.334

Sloterdijk, P. (2010). En el mundo interior del capital: para una teoría filosófica de la globalización. Siruela.

Stiglitz, J. (2002). El malestar en la globalización. Taurus.

Taussig, M. (2002). Chamanismo, colonialismo y el hombre salvaje. Norma.

Taylor, C. (2004). Modern Social Imaginaries. Duke University Press.

Taylor, C. (2006). Fuentes del yo. La construcción de la identidad moderna. Paidós.

Tepecikliolu, A. (2016). An analysis of the world society conceptualization in the English School. Ege Academic Review, 16 (4), 733-743.

Tilly, C. (2007). Guerra y construcción de estado como crimen organizado. Relaciones Internacionales, 5, I-26

Zea, L. (1978). Filosofía de la historia américana. Fondo de Cultura Económica.

Zea, L. (2010). La filosofía latinoaméricana como filosofía sin más. Siglo XXI. 


\section{RELACIONES INTERNACIONALES}

Revista académica cuatrimestral de publicación electrónica Grupo de Estudios de Relaciones Internacionales (GERI)

Universidad Autónoma de Madrid, España

https://revistas.uam.es/relacionesinternacionales

ISSN 1699 - 3950

f facebook.com/RelacionesInternacionales

3. twitter.com/RRInternacional

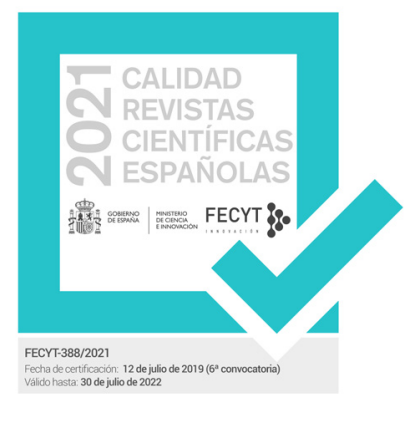

